Rel i abi I ity and fal I experi ence di scri mi nat i on of cross step moving on four spots test in the el derly

\begin{tabular}{|l|l|}
\hline 著者 & $\begin{array}{l}\text { Demur a Shi ni chi , Sat o Susum, M t sumor i } \\
\text { At suko, Sat o Toshi r o }\end{array}$ \\
\hline $\begin{array}{l}\text { j our nal or } \\
\text { publ i cat i on t i t l e }\end{array}$ & $\begin{array}{l}\text { Ar chi ves of Phy si cal Medi ci ne and } \\
\text { Rehabi I i t at i on }\end{array}$ \\
\hline vol une & 94 \\
\hline number & 7 \\
\hline page r ange & $1312-1319$ \\
\hline year & $2013-07-01$ \\
\hline URL & ht t p: //hdl . handl e. net /2297/35112 \\
\hline
\end{tabular}




\title{
Reliability and fall experience discrimination of Cross Step moving on Four spots Test in the elderly
}

\begin{abstract}
Objectives: To examine the reliability and fall experience discrimination of the Cross Step moving on Four spots Test (CSFT) including a rapid crossover steps and the relationship between the CSFT scores and the fall-related physical function.

Design: The reliability of the CSFT was examined in a test-retest format with the same tester. Fall history, fall risk, fear of falling, activities of daily living (ADL), and various physical parameters were measured for all participants.

Setting: A community center and university medical school in Japan

Participants: Elderly community-dwelling subjects aged 65-94 years living independently (62 men and 471 women)

Interventions: Not applicable
\end{abstract}

Main Outcome Measures: Time to complete all the CSFT steps required, fall risk score, ADL score, and fall-related physical function (isometric muscle strength: toe grip, plantar flexion, knee extension, hip flexion, hand grip; balance: one-leg standing time with eyes open, functional reach test using an elastic stick; and gait: 10-m maximal walking speed).

Results: The trial-to-trial reliability test indicated good reliability of the CSFT in both sexes (intraclass correlation coefficient $=0.833$ in men, 0.825 in women). However, trial-to-trial errors increased with increase in the CSFT values in both sexes. Significant correlations were observed between the CSFT values and scores for most fall-related physical function tests in both sexes. However, the correlation coefficient for all significant correlations was $<0.5$. Two-way analysis of variance (sex $\times$ fall experience) revealed that the fall experience is a 
significant factor affecting the CSFT values; the values in fallers were significantly lower than those in nonfallers. The odds ratios in logistic regression analysis were significant in both sexes (men: 1.35, women: 1.48). As determined by Youden's index, the optimal cutoff value for identifying fall experience was $7.32 \mathrm{~s}$, with an area under the curve of 0.676.

Conclusion: The CSFT can detect fall experience and is useful in the evaluation of different fall-related physical functions, including muscle strength, balance, and mobility.

Key words: fall risk, older person, balance, crossover step

List of abbreviations

CSFT: Cross-Step moving on Four spots Test

ADL: activities of daily living

DFRA: Demura's Fall Risk Assessment

ICC: intraclass correlation coefficients

ANOVA: analysis of variance

95\%CI: 95\% confidence intervals

ROC: Receiver operating characteristic

In most countries with an aging population, maintenance of independence in the elderly is important for avoiding excessive increase in national medical expenses. Physical and psychological trauma, loss of independence, or even death can occur in this population because of falls ${ }^{1,2}$. Falling in the elderly is a serious problem closely related to decreased quality of life. Thirty percent of all community-dwelling individuals over 65 years of age fall at least once each year $^{1}$. Of the elderly who have experienced falls, $31 \%-48 \%$ have a fear of falling again ${ }^{3-5}$ and 
$19 \%-26 \%$ choose to decrease activity levels to avoid falling ${ }^{6,7}$. Fall injuries occur in $46 \%-60 \%$ of falls ${ }^{4,8}$.

Multiple risk factors such as physical function levels, visual acuity and hearing ability, disease and disorders, cognitive function, medications, home environment, and age are related to falls ${ }^{3,9}$. Improving fall-related physical function, which is manifested by leg strength, balance, and mobility, has been reported to be effective in preventing falls ${ }^{2,10}$. These parameters can be improved by increased physical activity and exercises designed specifically for these individuals.

Improved physical function enhances the fixed-support strategy, which contributes to fall prevention by maintaining the center of gravity on a supportive base using ankle, knee, and hip muscles ${ }^{11}$. Falls are triggered when a large disturbance in sensory input disrupts body stability. Even physically fit elderly individuals are prone to falls because of conditions such as slipping or tripping ${ }^{12,13}$. Previous studies have attributed $40 \%-60 \%$ of falls to tripping ${ }^{14}$ and $10 \%-15 \%$ to slipping ${ }^{8,15}$. These results suggest that the ability to use a reactive change-in-support stepping strategy may be useful in averting falls in many cases ${ }^{11}$. The elderly are more severely affected by smaller disturbances than younger individuals ${ }^{11}{ }_{\text {._Adequate }}$ stepping strategies for averting a fall are even more important in the elderly ${ }^{11}$; this requires control of body sway and rapid adjustment of the center of gravity in the direction deviating from the supportive base ${ }^{16}$. Therefore, a rapid step test may be useful for evaluating the ability to avert a fall. The Rapid Stepping Test ${ }^{17-19}$, the Alternate Step Test ${ }^{20}$, and the Choice Stepping Reaction Time Test ${ }^{21}$ were previously developed to evaluate the fall avoidance ability in clinical settings.

However, an adequate stepping strategy should include consideration of the motion characteristics of the elderly in addition to the speed of stepping. Stepping in lateral directions is 
more difficult for the elderly than stepping forward and backward ${ }^{11}$. Lateral steps can be categorized as side steps and crossover steps. The former involves stepping to the side, and the latter involves stepping with one leg hooked behind the other ${ }^{22}$. Both stepping strategies are easy for the young, but the elderly are more likely to take crossover steps ${ }^{23}$. The side step requires rapid perception of displacement of the center of gravity before stepping and returning balance to the opposite side. This step is difficult for the elderly because of their delayed perception reactions ${ }^{23,24}$. Moreover, rapid crossover steps are also difficult because the legs become tangled ${ }^{11}$.

The Maximal Stepping Test requires rapid stepping on the spot with maximum effort ${ }^{17-19,25}$, and the Four Square Step Test involves rapid step movements, involving three or four changes in one direction ${ }^{16,26,27}$. In both of these tests,_subjects are required to step quickly on preassigned spots. However, these tests do not require crossover stepping. In this study, we evaluated a newly developed, clinically useful, rapid step test that includes crossover steps - the Cross Step moving on Four spots Test (CSFT). The purposes of this study were to examine the reliability of the CSFT, to clarify the relationship between the CSFT and fall-related physical function, and to confirm the ability of the CSFT to discriminate between fallers and nonfallers.

\section{METHODS}

\section{Participants}

Sixty-two men and 471 women aged 65-94 years living independently in the community participated in this study. They were selected on the basis of the following criteria: ambulation without walking aid and absence of cognitive impairment determined by the Mini-Mental State Examination (cutoff value: $>23$ point) ${ }^{28}$. All participants spent time outdoors at least 1 day/week and participated in a weekly or biweekly education program or 
light exercise program. To examine the validity of the CSFT in subjects with previous experience of falling, participants were divided into two groups - those who had experienced one or more falls in the previous year (fallers) and those who had not (nonfallers). Table 1 shows characteristics of the participants. No significant differences in age, height, or weight were noted between the fallers and nonfallers in either sex. Informed consent was obtained from each participant after full explanation of the experimental project and its procedure. This study was approved by the Ethics Committee on Human Experimentation of Faculty of Education, Kanazawa University.

\section{Procedures}

Prior to testing, all participants obtained medical clearance and approval from their primary care physicians to participate in this study. Participants visited the community center and completed a questionnaire to provide details of self-perceived activities of daily living (ADL) levels ${ }^{29}$. Fall risk was assessed using Demura's Fall Risk Assessment (DFRA) ${ }^{30,31}$, which was also used to record self-reported fall history over the previous year. This information was used to classify participants according to fall experience. The DFRA comprises questions regarding previous fall experience and 50 other fall risk assessment items representing the following 5 risk factors: potential for falling, physical function, disease and physical symptoms, environment, and behavior and character ${ }^{30,31}$. A fall was defined as any unintentional contact of a body part other than the feet onto any low surface such as the ground $^{1,3}$.

The participants performed the steps required in the CSFT. Isometric muscle strength tests (toe grip, plantar flexion, knee extension, hip flexion, and hand grip) and the functional reach test using an elastic stick were also performed. One-leg support time with open eyes was 
measured to determine the participants' ability to balance, and 10-m maximal walking speed was also measured. All the participants were permitted to rest as required during testing. The test order was randomly assigned to each participant.

\section{CSFT}

The apparatus for conducting the CSFT comprised four square sheets $(32 \mathrm{~cm} \times 32 \mathrm{~cm})$ capable of measuring foot contact time at a sampling frequency of $100 \mathrm{~Hz}$ and connected to a personal computer that saved the generated data (Fig. 1, left panel) ${ }^{a}(\mathrm{~S}-08069)$. The sheets were set in a cruciform pattern with an empty center position. Figure 1 (right panel) shows the stepping protocol of the CSFT. Participants stood in square number I facing square number III as shown in Figure 1. The aim was to step as fast as possible into each square in the sequence shown in Figure 1 (right panel) as follows: II, III, IV, I, IV, III, II, and I. This sequence required each participant to complete two rounds - one counterclockwise and one clockwise. The participants were instructed to face forward during stepping and to step on each square using a predefined pattern for moving the feet (Fig. 1, right panel). The CSFT included crossover steps (protocols number 3, 4, 8, and 9).

Prior to completing the CSFT, the participants were allowed a practice trial to ensure complete understanding of the step sequence. The CSFT was performed twice with a 3-min interval for rest. The total time to complete all the steps was measured. In all analyses except the trial-to-trial reliability analysis, the best times were adopted. The round was repeated if a participant failed to complete the sequence successfully, stepped off the sheet, or lost balance during the sequence. A tester stood behind each participant during testing to protect them from falling if they lost their balance and to ensure correct performance of all the sequences. The entire test, including instruction time, practice trial, and rest interval, took less than 8 min 
to complete.

\section{Physical function tests}

Physical function tests were performed to measure muscle strength, balance ability, and gait, which are related to mobility and posture control. Tests of isometric muscle strength included the toe grip, plantar flexion, knee extension, hip flexion, and hand grip tests. Balance was tested by measuring one-leg standing time with eyes open and performing the functional reach test using an elastic stick. Gait was assessed by analyzing 10-m walking time at maximal speed. Toe grip, plantar flexion, knee extension, hip flexion, and hand grip were measured using the following dedicated instruments: the Toe Grip Dynamometer ${ }^{\text {a }}$ (TKK3362), Plantar Flexion Measurement ${ }^{\mathrm{a}}$ (S-08093), Tension Meter D ${ }^{\mathrm{a}}$ (TKK5710e), Hand-Held Dynamometer ${ }^{\mathrm{b}}$ ( $\mu$ TAS F-1), and Grip Dynamometer ${ }^{\mathrm{a}}$ (Grip-D TKK5401), respectively. Plantar flexion was measured with participants in a long sitting position on the floor, whereas the other parameters were measured with participants sitting on chairs. All muscle strength tests were measured twice in both legs, and mean maximum values for each joint on both sides were used for the analysis. Functional reach was measured using an elastic stick $^{32}$. Each participant extended the dominant hand to the farthest possible point forward in an upright posture while touching the top of the elastic stick fixed at a height on the wall at the horizontal level of the acromion of the dominant side. They were required to push and shorten the elastic stick with the extended dominant hand. The length of the elastic stick was then measured.

\section{Data analyses}

The intraclass correlation coefficient (ICC) was used to evaluate the trial-to-trial reliability 
of the CSFT. One-way repeated measures analysis of variance (ANOVA) was used to determine mean differences between the CSFT trials. The Bland-Altman plot was used to evaluate systematic errors between the CSFT trials. Pearson's correlation coefficient was calculated to reveal relationships among the CSFT values, ADL scores, and leg muscle function. The following methods of data analyses were used to determine the validity of the CSFT. Two-way ANOVA (sex $\times$ fall experience) using Type III Sums of Squares was used to compare mean differences in the CSFT scores between fallers and nonfallers. Logistic regression analysis with $95 \%$ confidence intervals $(95 \% \mathrm{CI})$ was used to compare data between the sexes, and the odds ratio was estimated to observe the possible associations between the fall experience (dependent variable) and scores on the CSFT (independent variable). Receiver operating characteristic (ROC) analysis was constructed for the CSFT scores. The results were used to calculate the cutoff values to predict the likelihood of falling. The cutoff value was determined by the highest Youden's index ( $\mathrm{J}$ statistic) according to the following formula: $\mathrm{J}=$ sensitivity + specificity $-1^{33}$. A probability level of 0.05 was indicative of statistical significance.

\section{RESULTS}

Of those who required additional trials, 85 participants (15.9\%) failed to complete the step sequence and $31(5.8 \%)$ lost their balance and stepped off the squares. All participants who lost balance during the CSFT failed in the crossover step phase. All participants completed the CSFT within four trials.

Table 2 shows the self-perceived ADL scores and fall risk scores in each group. Table 3 shows the trial-to-trial reliability of the CSFT by sex. ICCs in men and women participants were 0.833 (95\%CI: $0.734-0.898)$ and 0.825 (95\%CI: 0.793-0.853), respectively. Significant 
differences were observed in the CSFT values between the trials, and the second trial was usually of shorter duration than the first. Cohen's effect sizes of trial differences were 0.40 in men and 0.27 in women. Figures 2 and 3 show the Bland-Altman plot of two CSFT trials by sex to confirm the presence or absence of systematic errors. In both sexes, as the CSFT value increased, the trial-to-trial error also increased.

Table 4 shows the correlation coefficients for CSFT and ADL scores and the results of muscle strength, balance, and mobility tests by sex. Significant correlations were observed between the CSFT values and the results of all tests in women. Significant correlations were observed between ADL scores, toe grip, one-leg standing time with open eyes, and 10-m maximal walking speed tests in men. However, the correlation coefficients for all the significant correlations were $<0.5$.

Table 5 shows the results of two-way ANOVA (sex $\times$ fall experience). No significant interaction and sex differences_were observed. Fall experience was identified as a significant factor. The CSFT values in fallers were significantly greater than those in nonfallers.

Significant partial regression coefficients were observed in the logistic regression analysis for both sexes. Odds ratios were also significant in both sexes (men: $1.35,95 \% \mathrm{CI}=1.00-1.81$, women: $1.48,95 \% \mathrm{CI}=1.31-1.68)($ Table 6$)$. According to Youden's index, the optimal cutoff value for identifying fall experience was $7.32 \mathrm{~s}$, with an area under the curve of 0.676 (Fig. 4).

\section{DISCUSSION}

The participants in this study lived independently in the community, did not use walking aids, and spent time outdoors at least 1 day every week. A self-perceived ADL score of 24 and below-mentioned points were used as a cutoff value to determine frailty ${ }^{29}$. In this study, 
$33.8 \%$ of men and $32.9 \%$ of women were classified as frail. According to the DFRA, the cutoff score determining high potential for falling was $>1$ point ${ }^{30,31}$. In this study, $55.5 \%$ of men and $48.8 \%$ of women were estimated to have high potential for falling. In addition, $27.4 \%$ of men and $21.9 \%$ of women participants reported having fallen in the previous year. This ratio was higher than that in a previous study of Japanese elderly people $(15.8 \%)^{34}$. However, all participants in this study completed the CSFT without physical aid, although some participants required additional trials because of a mistake in the step sequence (15.9\%) or loss of balance (5.8\%). All participants who lost balance during the CSFT failed in the crossover step phase. Although crossover steps may be difficult for the elderly, those who live independently should be able to attempt the CSFT as well as any other physical function test. The reliability of the CSFT as a test of stepping ability was established in both sexes (ICC = 0.833 in men, 0.825 in women). Demura et al. ${ }^{26}$ reported ICC values of $>0.9$ for the Maximal Stepping Test, which evaluated stepping on a preassigned spot as quickly as possible. Hashidate et al. ${ }^{27}$ proposed a modified step test to measure the time required for repeated lateral stepping ( 5 times) on $10 \mathrm{~cm}$-high plates and reported ICCs of $>0.9$ in any direction. Moreover, Shin and Demura ${ }^{35}$ reported an ICC of 0.73 for the time difference between foot contact on a preassigned spot and a stipulated tempo of 40 beats $/ \mathrm{min}$. The reliability values of the step parameters in the present study were similar to or slightly lower than the values in these reports. The discrepancy may be due to the high level of difficulty of the CSFT compared with that for the tests used in previous studies. Participants were prone to lose balance while performing the CSFT because they were required not only to step rapidly in several directions but also to take crossover steps. The trial-to-trial error revealed by the Bland-Altman plot tended to be greater in the participants with inferior CSFT ability in both sexes. An additional analysis was conducted to determine ICCs in a subgroup comprising 
participants with larger CSFT values than the mean. ICCs of men and women in this subgroup were 0.756 (95\%CI: $0.536-0.880)$ and 0.781 (95\%CI: $0.715-0.833)$, respectively. These values were slightly lower than the ICCs in the group as a whole in both sexes. In addition, for frail elderly people who are not used to taking crossover steps in daily life, stability of posture takes precedence. These participants prioritized stepping carefully over stepping rapidly so as to not lose their balance and fall. The fear of falling was reported in $14.5 \%$ of men and $17.0 \%$ of women in this study. This fact may have affected the reliability of the results of the CSFT compared with those of other step tests.

Although participants in this study were allowed a practice trial to ensure complete understanding of the step sequence prior to testing, significant differences were found between trials. The practice effect was small, but definitely noticeable. Practice trials were not performed as fast as possible because their purpose was to convey the aim of test and the step sequence. In the first trial in which participants were asked to perform with maximal effort, the participants (especially those for whom the CSFT was significantly challenging) may have attached more importance to maintaining stability because of the fear of falling than to performing the steps correctly. In future studies using the CSFT, the test procedure should be modified to involve a practice trial with maximal effort or repetition of the trial three times or more.

Rapid crossover stepping is considered to be important for avoiding falls. In crossover stepping, the body position must change suddenly in an unstable direction, which rarely occurs under usual walking conditions. Difficult movements in addition to maintaining basic physical function (leg strength, balance, and mobility) related to fall prevention are demanded of elderly participants for the CSFT to be successfully performed. For this reason and because it includes crossover steps, the CSFT bears little resemblance to existing basic physical 
function tests. Although significant correlations were found between the CSFT scores and all parameters in women as well as with ADL scores, toe grip, one-leg standing with open eyes, and 10-m maximal walking speed in men, the correlations were weak.

Many researchers have examined the relationships between falling, speed, and stability of gait in elderly individuals ${ }^{36-38}$. Kim et al. ${ }^{39}$ reported that elderly individuals with fall experience are more unstable in gait than those without such experience. Lee et al. ${ }^{40}$ reported differences between groups with or without a previous fall in tests, including a $2.45-\mathrm{m}$ round-trip walk, 10-m gait time, cadence, and one-leg standing time. On the other hand, a relationship between functional reach and one-leg standing with open eyes ${ }^{41,42}$ was reported as fair or good in the elderly, but relationships between muscle strength and balance ${ }^{41}$ and those between muscle strength and walking speed ${ }^{9,36,41}$ were reported as poor. Summarizing the results of these previous studies, physical functions such as strength, balance, and mobility reflect fall risk, but the relationships among these parameters are not always strong. In this study, correlations among strength, balance, and mobility were $<0.39$ in men and $<0.50$ in women, and the CSFT values were weakly correlated with these parameters.

Fall prevention is an essential part of well-rounded exercise programs comprising strength, balance, and mobility training and is more effective for elderly people than performing single exercises in isolation ${ }^{43}$. The present results suggested that rapid crossover stepping should be added to existing fall prevention exercise programs.

Differences between fallers and nonfallers were found in the CSFT values in this study. Significant odds ratios were also observed in the results of the logistic regression analysis in both sexes, and the validity of the CSFT to identify fallers and nonfallers was confirmed. The odds ratios suggested that an increase of $1 \mathrm{~s}$ in the CSFT value increased the possibility with fall history by 1.35 (95\%CI: $1.00-1.81)$ in men and by 1.48 (95\% CI: $1.31-1.68)$ in women. 
The ROC analysis in this study identified a cutoff value in the CSFT score of $7.32 \mathrm{~s}$ for distinguishing between fallers and nonfallers.

Hilliard et al. ${ }^{44}$ reported that although approximately $70 \%$ of the elderly can take crossover steps in the lateral direction when experiencing a disturbance, fallers tended to take multiple steps, including crossover steps. Moreover, the elderly who used multiple steps to recover balance were 6.2 times more likely to fall than those who did not always use them ${ }^{44}$. Maki and McIlroy ${ }^{11}$ reported that many elderly tend to tumble over their own feet during crossover stepping regardless of leg strength and balance ability when experiencing floor oscillation. The present study suggested that the preliminary validity of CSFT may be useful in predicting falling potential in the elderly because it requires taking rapid crossover steps without losing balance which is critical for fall-averting ability and identifying fall history within 1 year.

\section{Study Limitations}

Generalization of the results should be done with caution. Because the total time to complete all the steps of the CSFT is minimal and the results can be measured using only a stopwatch, the CSFT will be easily applicable in clinical settings. On the other hand, when considering the ability of participants to perform crossover stepping, the crossover step time may be better evaluated separate from the total time. This will need to be examined in a future study. To evaluate fall-averting ability, the most valid method is the one that best simulates conditions that induce falling in a laboratory setting, such as the floor oscillation test performed by Maki and McIlroy ${ }^{11}$. However, this method cannot be widely applied because of the high risk of injury to the elderly. Future studies should examine the relationship between fall-averting ability measured under simulated conditions and those measured using CSFT values. In 
addition, Rogers and Mille ${ }^{22}$ emphasized the importance of enhancing hip abduction and adduction strength for stability during lateral disturbance. Training for the purpose of improving CSFT values may be possible through exercise including crossover steps or resistance training of the gluteus medius and adductor muscles involved in hip abduction and adduction, respectively.

\section{CONCLUSION}

The reliability of the CSFT was demonstrated in this study. A modified test procedure may include a practice trial requiring maximum effort after and repeated performance of three repetitions or more. The CSFT evaluated various parameters of fall-related physical function on the basis of tests for muscle strength, balance, and mobility. The difference of CSFT between fallers and nonfallers was detected, and the odds ratios suggested that an increase of $1 \mathrm{~s}$ in CSFT increased the possibility of having a fall history of 1.35 (95\%CI: $1.00-1.81)$ in men and 1.48 (95\%CI: $1.31-1.68)$ in women. The cutoff value for distinguishing between fallers and nonfallers was $7.32 \mathrm{~s}$.

\section{REFERENCES}

1. Lord SR, Dayhew J. Visual risk factors for falls in older people. J Am Geriatr Soc. 2001; 49(5): 508-15.

2. Oliver D. Older people who fall: why they matter and what you can do. Br J Community Nurs. 2007; 12(11): 500-7.

3. Tinetti ME, Speechley M, Ginter SF. Risk factors for falls among elderly persons living in the community. N Engl J Med. 1988; 319(26): 1701-7.

4. Tinetti ME, Mendes de Leon CF, Doucette JT, Baker DI. Fear of falling and fall-related 
efficacy in relationship to functioning among community-living elders. J Gerontol. 1994; 49(3): M140-7.

5. Vellas BJ, Wayne SJ, Romero LJ, Baumgartner RN, Garry PJ. Fear of falling and restriction of mobility in elderly fallers. Age Ageing. 1997; 26(3): 189-93.

6. Nevitt MC, Cummings SR, Hudes ES. Risk factors for injurious falls: a prospective study. J Gerontol. 1991; 46(5): M164-70.

7. Tinetti ME, Powell L. Fear of falling and low self-efficacy: a case of dependence in elderly persons. J Gerontol. 1993; 48 Spec No:35-8.

8. Hill K, Schwarz J, Flicker L, Carroll S. Falls among healthy, community-dwelling, older women: a prospective study of frequency, circumstances, consequences and prediction accuracy. Aust N Z J Public Health. 1999; 23(1): 41-8.

9. Leipzig RM, Cumming RG, Tinetti ME. Drugs and falls in older people: a systematic review and meta-analysis: I. Psychotropic drugs. J Am Geriatr Soc. 1999; 47(1): 30-9.

10. Baczkowicz D, Szczegielniak J, Proszkowiec M. Relations between postural stability, gait and falls in elderly persons-preliminary report. Ortop Traumatol Rehabil. 2008; 10(5): 478-85.

11. Maki BE, McIlroy WE. Control of rapid limb movements for balance recovery: age-related changes and implications for fall prevention. Age Ageing. 2006; 35 Suppl 2: ii12-8.

12. Berg WP, Alessio HM, Mills EM, Tong C. Circumstances and consequences of falls in independent community-dwelling older adults. Age Ageing. 1997; 26(4): 261-8.

13. Topper AK, Maki BE, Holliday PJ. Are activity-based assessments of balance and gait in the elderly predictive of risk of falling and/or type of fall? J Am Geriatr Soc. 1993; 41(5): 479-87. 
14. Blake AJ, Morgan K, Bendall MJ, Dallosso H, Ebrahim SB, Arie TH, Fentem PH, Bassey EJ. Falls by elderly people at home: prevalence and associated factors. Age Ageing. 1988; 17(6): 365-72.

15. Cumming RG, Klineberg RJ. Fall frequency and characteristics and the risk of hip fractures. J Am Geriatr Soc. 1994; 42(7): 774-8.

16. Dite W, Temple VA. A clinical test of stepping and change of direction to identify multiple falling older adults. Arch Phys Med Rehabil. 2002; 83: 1566-71.

17. Hill K, Bernhardt J, McGann A, Maltese D, Berkovits D. A new test of dynamic standing balance for stroke patients: reliability, validity and comparison with healthy elderly. Physiother Can. 1997; 48: 257-62.

18. Cho BL, Scarpace D, Alexander NB. Tests of stepping as indicators of mobility, balance, and fall risk in balance-impaired older adults. J Am Geriatr Soc. 2004; 52(7): 1168-73.

19. Demura S, Yamaji S, Kitabayashi T. Gender and age-related differences of dynamic balancing ability based on various stepping motions in the healthy elderly. J Hum Ergol (Tokyo). 2005; 34(1-2): 1-11.

20. Tiedemann A, Shimada H, Sherrington C, Murray S, Lord S. The comparative ability of eight functional mobility tests for predicting falls in community-dwelling older people. Age Ageing. 2008; 37(4): 430-5.

21. Lord SR, Fitzpatrick RC. Choice stepping reaction time: a composite measure of falls risk in older people. J Gerontol A Biol Sci Med Sci. 2001; 56(10): M627-32.

22. Rogers MW, Mille ML. Lateral stability and falls in older people. Exerc Sport Sci Rev. 2003; 31(4): 182-7.

23. Patton JL, Hilliard MJ, Martinez K, Mille ML, Rogers MW. A simple model of stability limits applied to sidestepping in young, elderly and elderly fallers. Conf Proc IEEE Eng 
Med Biol Soc. 2006; 1: 3305-8.

24. Mille ML, Johnson ME, Martinez KM, Rogers MW. Age-dependent differences in lateral balance recovery through protective stepping. Clin Biomech (Bristol, Avon). 2005; 20(6): $607-16$

25. Demura S, Yamada T, Shin S. Age and sex differences in various stepping movements of the elderly. Geriatr Gerontol Int. 2008; 8: 180-7.

26. Medell JL, Alexander NB. A clinical measure of maximal and rapid stepping in older women. J Gerontol A Biol Sci Med Sci. 2000; 55(8): M429-33.

27. Hashidate H, Uchiyama Y, Shiomi T. Development of modified step test as dynamic balance assessment: reliability and validity. Rigakuryoho Kagaku. 2003; 19: 55-9.

28. Folstein MF, Robins LN, Helzer JE. The Mini-Mental State Examination. Arch Gen Psychiatry. 1983; 40(7): 812.

29. Ministry of Education, Culture, Sports, Science and Technology, in Japan (2000) Guideline for new physical fitness test. [http://www.mext.go.jp/a_menu/sports/stamina/03040901.htm]

30. Demura S, Sato S, Shin S, Uchiyama M. Setting the criterion for fall risk screening for healthy community-dwelling elderly. Arch Gerontol Geriatr. 2012; 54(2): 370-3.

31. Demura S, Sato S, Yamaji S, Kasuga K, Nagasawa Y. Examination of validity of fall risk assessment items for screening high fall risk elderly among the healthy community-dwelling Japanese population. Arch Gerontol Geriatr. 2011; 53(1): e41-5.

32. Demura S, Yamada T. Simple and easy assessment of falling risk in the elderly by functional reach test using elastic stick. Tohoku J Exp Med. 2007; 213: 105-11.

33. Bewick V, Cheek L, Ball J. Statistics review 13: receiver operating characteristic curves. Crit Care. 2004; 8(6): 508-12. 
34. Yokoya T, Demura S, Sato S. Fall risk characteristics of the elderly in an exercise class. J Physiol Anthropol. 2008; 27(1): 25-32.

35. Shin S, Demura S. Effective tempo of the step test for dynamic balance ability in the elderly. J Physiol Anthropol. 2007; 26(6): 563-67.

36. Baczkowicz D, Szczegielniak J, Proszkowiec M. Relations between postural stability, gait and falls in elderly persons-preliminary report. Ortop Traumatol Rehabil. 2008; 10: 478-85.

37. Smeesters C, Hayes WC, McMahon TA. Disturbance type and gait speed affect fall direction and impact location. J Biomech. 2001; 34(3): 309-17.

38. van Iersel MB, Olde Rikkert MG, Borm GF. A method to standardize gait and balance variables for gait velocity. Gait Posture. 2007; 26(2): 226-30.

39. Kim H, Yoshida H, Suzuki T, Ishizaki T, Hosoi T, Yamamoto S, Orimo H. The relationship between fall-related activity restriction and fitness in elderly women. Nippon Ronen Igakkai Zasshi. 2001; 38: 805-11. [In Japanese]

40. Lee S, Kim S, Park Y, Kim J. Effects of falling experience to gait pattern, functional fitness and leg strength of elderly women. Korean J Phys Educ. 2007; 46: 369-78. [In Korean]

41. Boulgarides LK, McGinty SM, Willett JA, Barnes CW. Use of clinical and impairment-based tests to predict falls by community-dwelling older adults. Phys Ther. 2003; 83(4): 328-39.

42. Desai A, Goodman V, Kapadia N, Shay BL, Szturm T. Relationship between dynamic balance measures and functional performance in community-dwelling elderly people. Phys Ther. 2010; 90(5): 748-60. Epub 2010 Mar 11.

43. Ouyang P, Yatsuya H, Toyoshima H, Otsuka R, Wada K, Matsushita K, Ishikawa M, 
Yuanying L, Hotta Y, Mitsuhashi H, Muramatsu T, Kasuga N, Tamakoshi K. Changes in activities of daily living, physical fitness, and depressive symptoms after six-month periodic well-rounded exercise programs for older adults living in nursing homes or special nursing facilities. Nagoya J Med Sci. 2009; 71(3-4): 115-26.

44. Hilliard MJ, Martinez KM, Janssen I, Edwards B, Mille ML, Zhang Y, Rogers MW. Lateral balance factors predict future falls in community-living older adults. Arch Phys Med Rehabil. 2008; 89(9): 1708-13.

\section{Suppliers}

a. Takei Scientific Instruments Co., Ltd, 619, Yashiroda, Akiha-Ku, Niigata, 956-0113, Japan. b. Anima Co., 3-65-1, Shimoishihara, Chofu, Tokyo, Japan. 
Table and figure legends

Table 1. Characteristics of the participants. M: mean; SD: standard deviation

Table 2. Self-perceived activities of daily living (ADL) scores and Demura's Fall Risk Assessment (DFRA) scores in men and women fallers and nonfallers

M: mean; SD: standard deviation

Table 3. Trial-to-trial reliability of the Cross Step moving on Four spots Test (CSFT)

M: mean; SD: standard deviation; ICC: intraclass correlation coefficient; CI: confidence intervals

Table 4. Correlations between CSFT with ADL scores and results of tests of physical parameters

$*: \mathrm{p}<0.05,{ }^{\mathrm{a}}: \mathrm{n}=62,{ }^{\mathrm{b}}: \mathrm{n}=471$

Table 5. Results of two-way analysis of variance (ANOVA) (sex $\times$ fall experience) of CSFT values (unit: seconds)

M: mean; SD: standard deviation; *: $\mathrm{p}<0.05$

Table 6. Odds ratios for fall experience according to the CSFT using a logistic regression model

B: partial regression coefficient, SE: standard error, CI: confidence intervals

Figure 1. Measurement device and stepping protocol of the Cross Step moving on Four spots Test (CSFT)

Figure 2. The Bland-Altman plot of CSFT results in men. SD: standard deviation

Figure 3. The Bland-Altman plot of CSFT results in women. SD: standard deviation

Figure 4. ROC analysis for the CSFT. AUC: area under the curve 
Table 1 Characteristics of the participants.

\begin{tabular}{|c|c|c|c|c|c|c|c|c|c|c|c|c|c|}
\hline & & \multicolumn{6}{|c|}{ Men } & \multicolumn{6}{|c|}{ Women } \\
\hline & & \multicolumn{2}{|c|}{ Total } & \multicolumn{2}{|c|}{ Faller } & \multicolumn{2}{|c|}{ Nonfaller } & \multicolumn{2}{|c|}{ Total } & \multicolumn{2}{|c|}{ Faller } & \multicolumn{2}{|c|}{ Nonfaller } \\
\hline & & M & SD & $\mathrm{M}$ & $\mathrm{SD}$ & M & SD & $\mathrm{M}$ & SD & M & SD & M & $\mathrm{SD}$ \\
\hline Number of participants & (n) & \multicolumn{2}{|c|}{62} & \multicolumn{2}{|c|}{17} & \multicolumn{2}{|c|}{45} & \multicolumn{2}{|c|}{471} & \multicolumn{2}{|c|}{103} & \multicolumn{2}{|c|}{368} \\
\hline Fear of falling & $(\%)$ & \multicolumn{2}{|c|}{$14.5 \%$} & \multicolumn{2}{|c|}{$5.9 \%$} & \multicolumn{2}{|c|}{$17.8 \%$} & \multicolumn{2}{|c|}{$17.0 \%$} & \multicolumn{2}{|c|}{$30.1 \%$} & \multicolumn{2}{|c|}{$13.3 \%$} \\
\hline Age & (years) & 76.2 & 7.3 & 76.6 & 8.7 & 76.0 & 6.8 & 76.2 & 5.8 & 76.6 & 5.7 & 76.1 & 5.8 \\
\hline Height & $(\mathrm{cm})$ & 160.8 & 7.2 & 161.0 & 6.7 & 160.7 & 7.4 & 147.6 & 5.7 & 148.2 & 5.7 & 147.4 & 5.6 \\
\hline Weight & (kg) & 59.9 & 9.9 & 60.1 & 10.8 & 59.9 & 9.7 & 50.0 & 9.1 & 50.7 & 7.5 & 49.8 & 9.5 \\
\hline
\end{tabular}

M: mean; SD: standard deviation 
Table 2 . Self-perceived activities of daily living (ADL) scores and Demura's Fall Risk Assessment (DFRA) scores in men and women fallers and nonfallers

\begin{tabular}{|c|c|c|c|c|c|c|c|c|c|c|c|c|}
\hline & \multicolumn{6}{|c|}{ Men } & \multicolumn{6}{|c|}{ Women } \\
\hline & \multicolumn{2}{|c|}{ Total } & \multicolumn{2}{|c|}{ Faller } & \multicolumn{2}{|c|}{ Nonfaller } & \multicolumn{2}{|c|}{ Total } & \multicolumn{2}{|c|}{ Faller } & \multicolumn{2}{|c|}{ Nonfaller } \\
\hline & $\mathrm{M}$ & SD & $\mathrm{M}$ & SD & $\mathrm{M}$ & SD & $\mathrm{M}$ & SD & $\mathrm{M}$ & $\mathrm{SD}$ & $\mathrm{M}$ & $\mathrm{SD}$ \\
\hline ADL score & 25.6 & 5.2 & 26.7 & 5.7 & 25.2 & 5.1 & 25.1 & 4.9 & 23.1 & 4.9 & 25.7 & 4.7 \\
\hline $\begin{array}{l}\text { Potential for falling score (point) } \\
\text { by DFRA }\end{array}$ & 0.8 & 0.8 & 0.8 & 0.9 & 0.8 & 0.8 & 0.7 & 0.8 & 1.2 & 0.8 & 0.5 & 0.7 \\
\hline
\end{tabular}

M: mean; SD: standard deviation 
Table 3 Trial-to-trial reliability of the Cross Step moving on Four spots Test (CSFT)

\begin{tabular}{|c|c|c|c|c|c|c|c|}
\hline & & FCS & (s) & & & & \\
\hline & $1 \mathrm{st}$ & rial & 2 nd & trial & $\mathrm{E}_{2}$ & ICC & $050 / C$ \\
\hline & $\mathrm{M}$ & SD & $\mathrm{M}$ & SD & & & \\
\hline Men & 7.6 & 2.9 & 6.6 & 2.1 & $49.4 *$ & 0.833 & $0.734-0.898$ \\
\hline Women & 7.2 & 2.4 & 6.4 & 2.0 & $293.9 *$ & 0.825 & $0.793-0.853$ \\
\hline
\end{tabular}

M: mean; SD: standard deviation; ICC: intraclass correlation coefficient; CI: confidence intervals 
Table 4 Correlations between CSFT with ADL scores and results of physical parameters

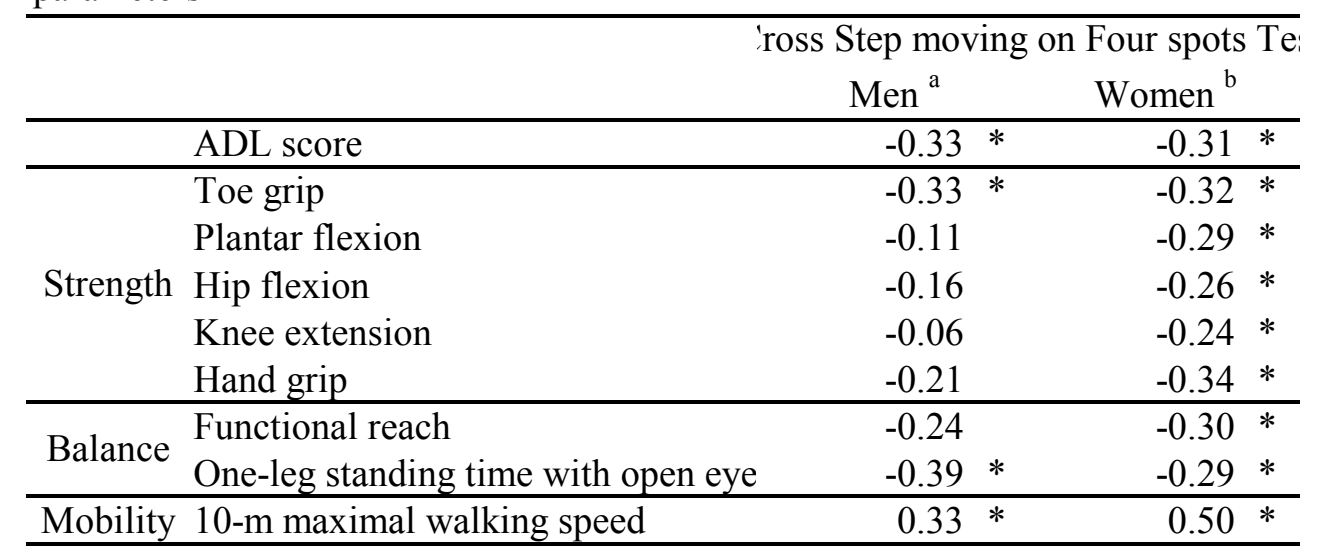

$*: \mathrm{p}<.05,{ }^{\mathrm{a}}: \mathrm{n}=62,{ }^{\mathrm{b}}: \mathrm{n}=471$ 
Table 5 Results of two-way analysis of variance (ANOVA) (sex $\times$ fall experience) of CSFTvalues (unit: seconds)

\begin{tabular}{|c|c|c|c|c|c|c|c|c|c|}
\hline & \multicolumn{3}{|c|}{ Faller } & \multicolumn{3}{|c|}{ Non-faller } & \multicolumn{3}{|c|}{ ANOVA F-value } \\
\hline & $\mathrm{n}$ & M & $\mathrm{SD}$ & $\mathrm{n}$ & M & SD & Sex & $\begin{array}{c}\text { Fall } \\
\text { experience }\end{array}$ & Interaction \\
\hline Men & 17 & 7.97 & 4.13 & 45 & 6.43 & 1.28 & 0.18 & $14.92 *$ & 0.02 \\
\hline Women & 103 & 7.85 & 4.80 & 368 & 6.20 & 1.80 & & & \\
\hline
\end{tabular}

M: mean; SD: standard deviation; *: $\mathrm{p}<.05$ 
Table 6 Odds ratios for fall experience according to the CSFT using a

logistic regression model

\begin{tabular}{llcrrrrrrr}
\hline & $B$ & SE & Wald & p & Odds ratio & \multicolumn{2}{c}{$95 \%$ CI } \\
\hline FCST & Men & 0.30 & 0.15 & 3.96 & 0.047 & 1.35 & 1.00 & -1.81 \\
& Women & 0.39 & 0.06 & 37.76 & 0.000 & 1.48 & 1.31 & -1.68 \\
\hline
\end{tabular}

$B$ : partial regression coefficient, SE: standard error, CI: confidence intervals 


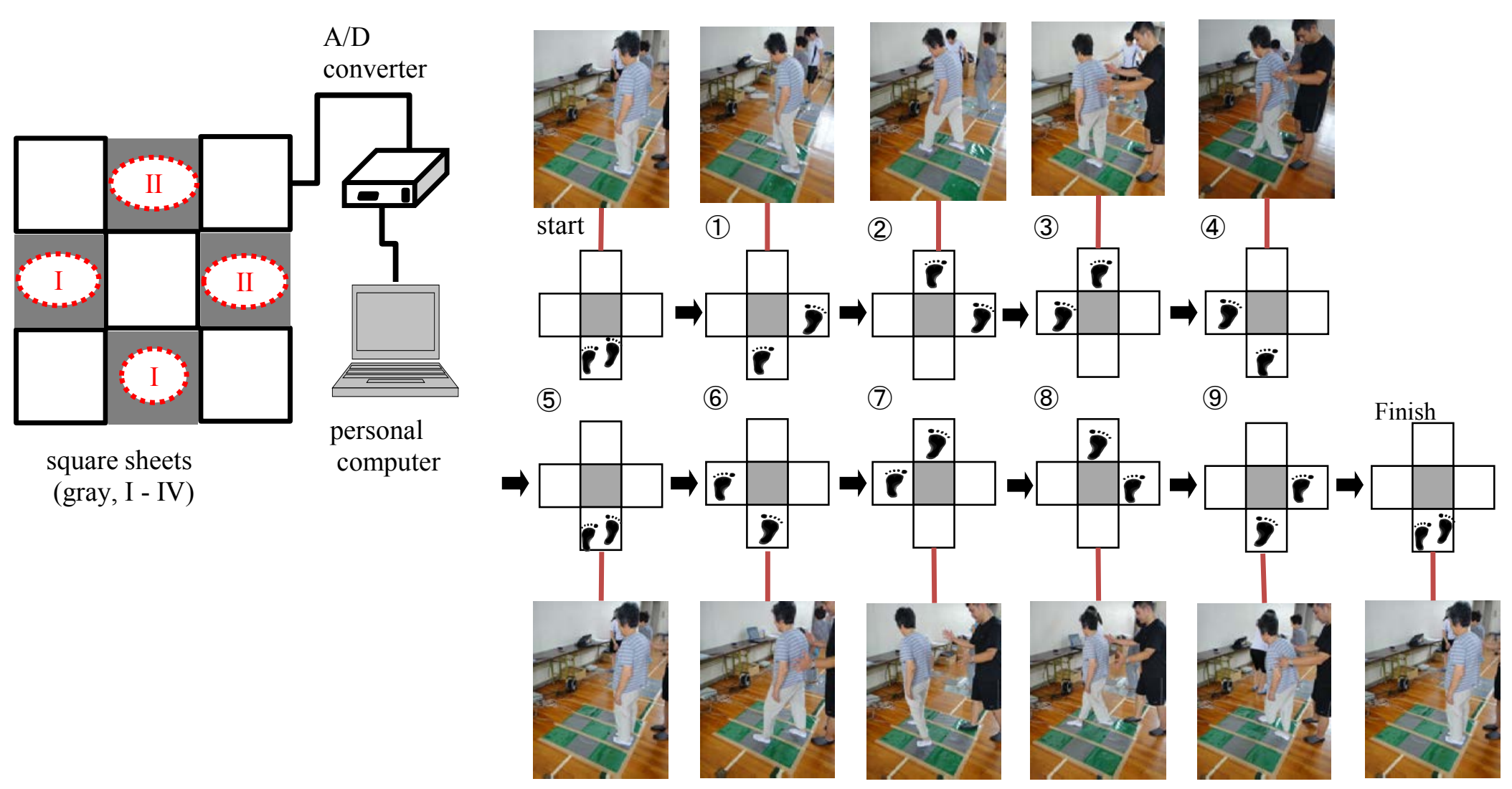

Figure 1 The measurement device and stepping protocol of Cross Step moving on Four spots Test 


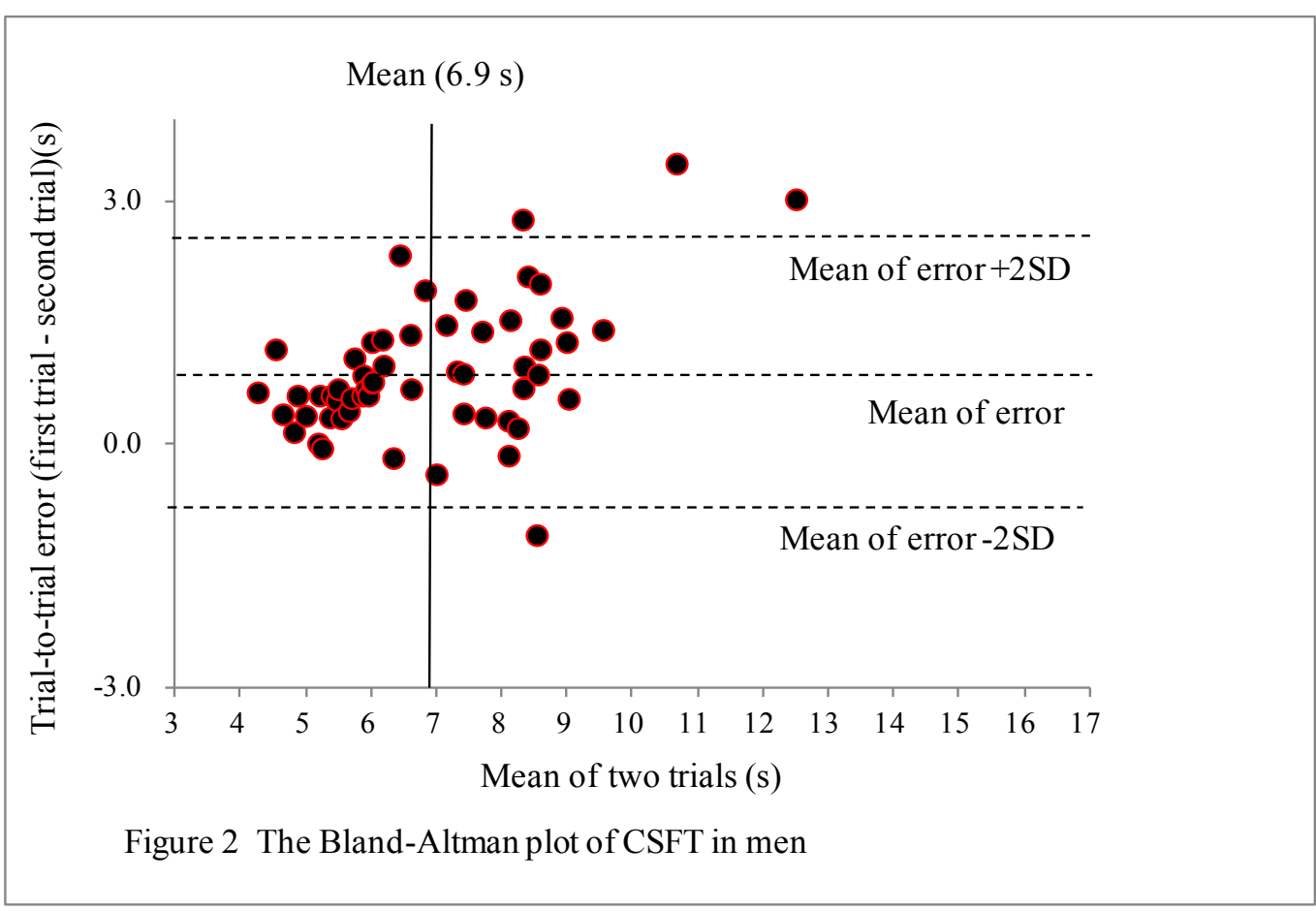




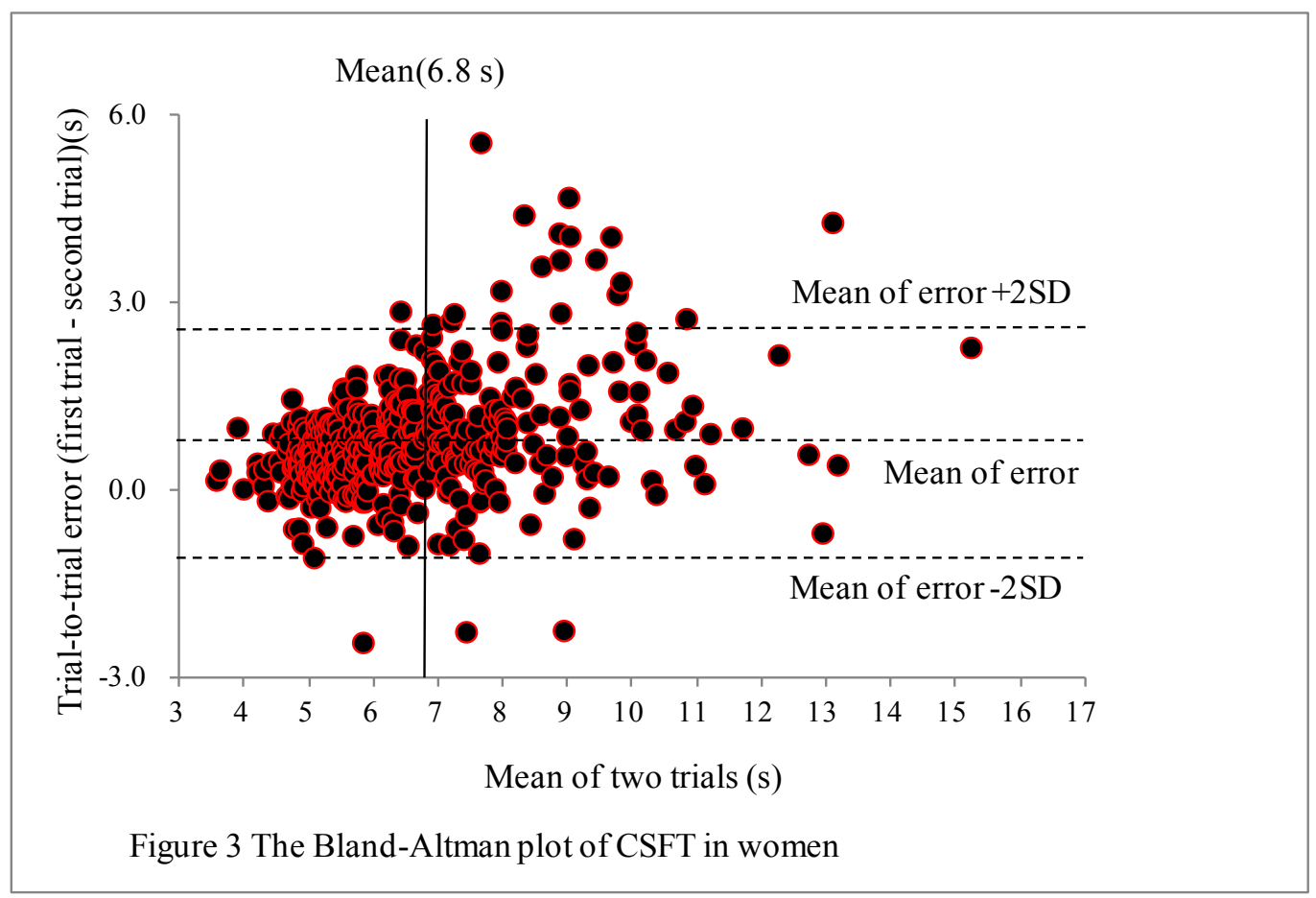




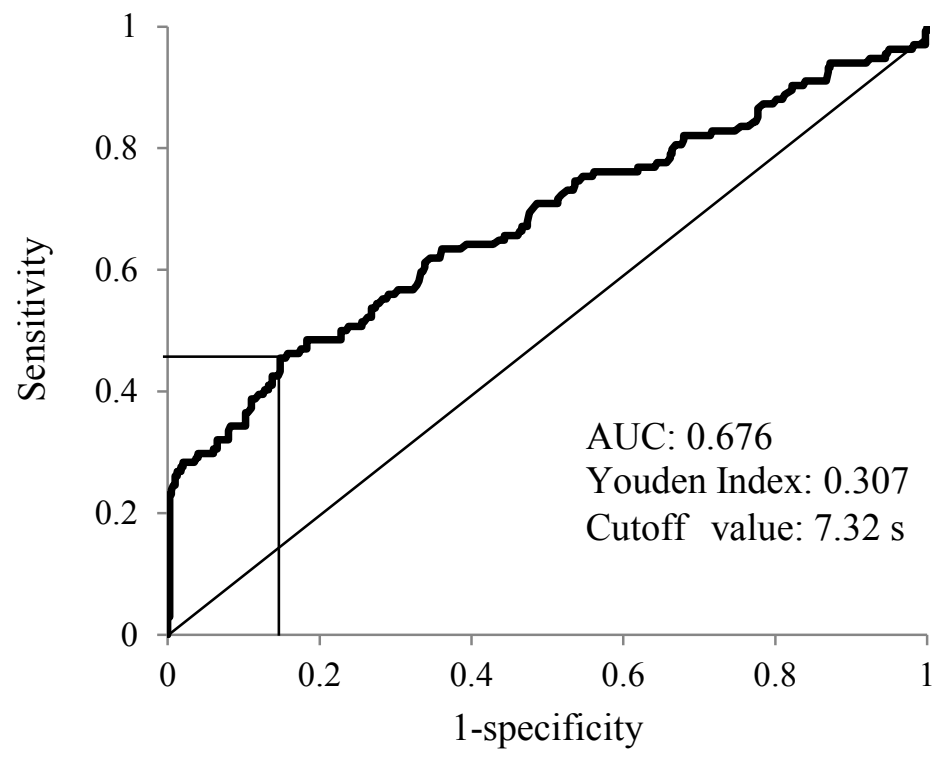

Figure 4 ROC analysis for the CSFT.

AUC: area under the curve 